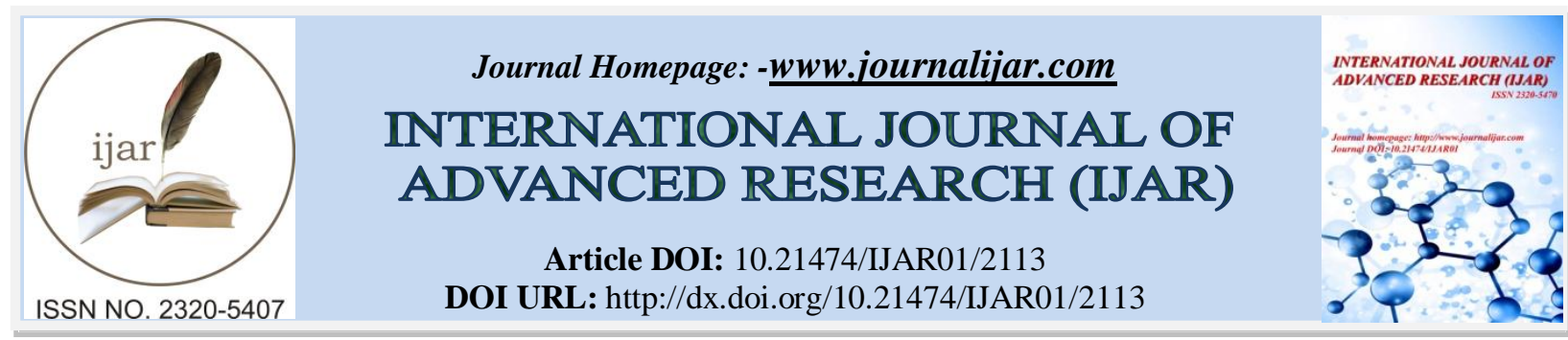

RESEARCH ARTICLE

\title{
STUDY OF CASTING DEFECTS AND THEIR REMEDIES - A REVIEW.
}

\author{
Mrs. Rashi P.Gaikwad ${ }^{1}$ and Mr. Pankaj A. Gaikwad ${ }^{2}$.
}

1. Assistant Professor' Mechanical Engineering, Indira College of Engineering, Pune.

2. Manager, Bajaj Auto Limited, Pune.

\section{Manuscript Info}

Manuscript History

Received: 25 September 2016

Final Accepted: 27 October 2016

Published: November 2016

Key words:-

Casting, Gating System and Melting and pouring.

\begin{abstract}
Casting is the one of the oldest manufacturing processes, and even today is the first step in manufacturing most product. In this process the material is first liquefied by proper heating and then this liquefied is poured into a previously prepared mould cavity where it is allowed to solidify and subsequently the product is taken out, cleaned and trimmed to the required shape.

During this process there are different critical factors which is contributing in large amount to the defects. These factors are:

1. Design of casting and pattern

2. Moulding sand and design of mould and core.

3. Metal composition.

4. Melting and pouring.

5. Gating system.
\end{abstract}

Copy Right, IJAR, 2016,. All rights reserved.

\section{Introduction:-}

Casting is the one of the oldest manufacturing processes, and even today is the first step in manufacturing most product. In this process the material is first liquefied by proper heating and then this liquefied is poured into a previously prepared mould cavity where it is allowed to solidify and subsequently the product is taken out, cleaned and trimmed to the required shape.

A pattern is the replica of the part to be cast and is use to prepare the mould cavity. Pattern are made of either wood or metal. When making a pattern it is best to taper the edges so that the pattern can be removed without breaking the mould. The patterns are then packed in sand with a binder, which helps to harden the sand into a semi-permanent shape. Once the sand mould is cured, the pattern is removed leaving a hollow space in the sand in the shape of the desired part. The pattern is intentionally made larger than the cast part to allow for shrinkage during cooling. Sand cores can then be inserted in the mould to create holes and improve the casting's net shape. [1]

The material for green sand mould is a mixture of sand, clay, water and some organic additives. A mould is an assembly of two or more metal blocks, or bonded refractory particles (sand) consisting of a primary cavity. The mould cavity holds the liquid material and essentially acts as a negative of the desired product. A proper care during melting is essential. The factors to be considered during melting include gases in metal, selection and control of scrap, flux, furnace, and temperature.

A good gating design ensures distribution of the metal in the mould cavity at a proper rate without excessive temperature loss, turbulence and entrapping gases and slags. Casting defects analysis is process of finding the root cause of occurrence of defects in the rejection of casting and taking necessary steps to reduce the defects and to 
improve the casting yield. Taguchi method is used for analysis casting defects like sand and mould related defects such as sand drop, bad mould, blow holes, cuts and washes.

\section{Causes And Remedies In Casting}

In this section we study the different types of defects in casting, and there origin and remedies. The defects may arise due to the defects in one or more of the following factors; [2][3]

Design of casting and pattern

1. Moulding sand and design of mould and core.

2. Metal composition.

3. Melting and pouring.

4. Gating system.

The most commonly encountered defects in the sand mould castings are:

1. Blow- It is a largely well - rounded cavity produced by the gases which displace the molten metal at the cope surface of a casting.

Generally occurs on the convex casting surface.

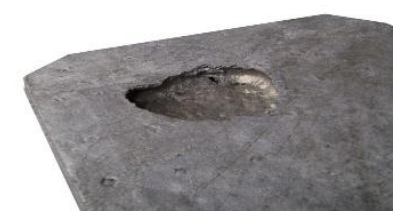

Causes-

\section{Figure 0-1 Blow Hole}

i. $\quad$ Excessive moisture content in the mould

ii. Rust and moisture in chill

iii. Cores are not sufficiently baked

iv. Excessive use of organic binder

v. Mould rammed very hard.

Remedy:

i. $\quad$ Can be avoided by having a proper venting and adequate permeability

ii. Controlled content of moisture and volatile constituents in the sand - mix.

iii. Ram the mould less.

2. Pin Holes- These are tiny blow holes.

Occur either at or just below the casting surface.

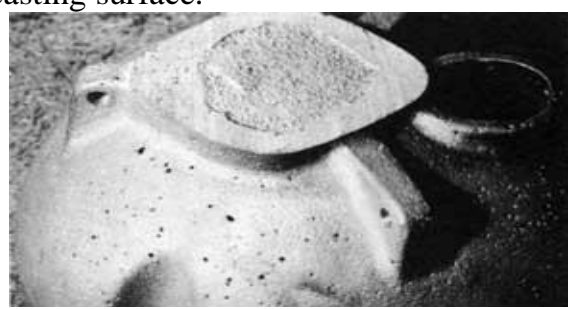

Figure 0-2 Pin Hole

Cause-

i. $\quad$ Occurs due to gas dissolved in the alloy \& the alloy not properly degasses.

Remedy-

i. Maintaining pouring temperature.

3. Drop- An irregularly - shaped projection on the cope surface of a casting is called drop. 


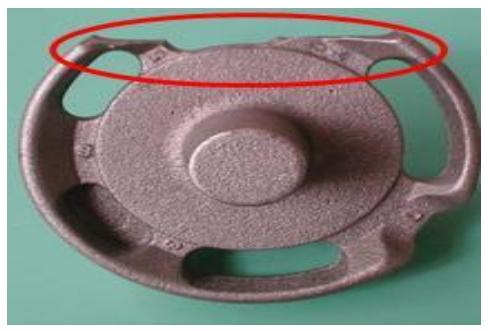

Cause-

Figure 0-3 Drop

i. By dropping of sand from the cope or other overhanging projections into the mould.

ii. Inadequate of reinforcement of sand and core projection.

Remedy -

i. An adequate strength of the sand and the use of gaggers can avoid the drops.

ii. Provide harder rammer.

iii. Increase green strength of green sand.

4. Dross- Lighter impurities appearing on the top surface of a casting.

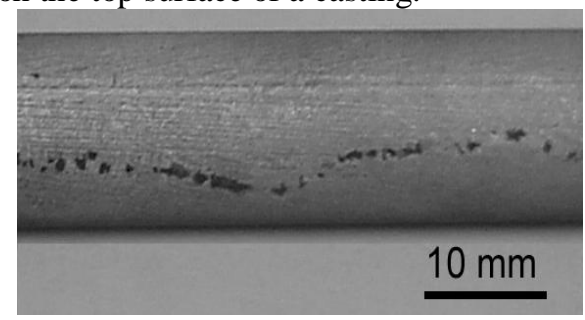

Cause -

Figure 0-4 Dross

i. $\quad$ Very high temperature.

ii. Composition of molten metal

Remedy-

i. $\quad$ Using strainer and skim bob at the pouring stage.

5. Dirt- sand particles dropping out of the cope get embedded on the top surface of the casting when removed leave small angular - holes.

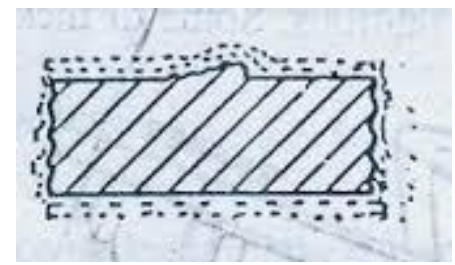

Figure 0-5 Dirt

Cause-

i. Hydrogen in the molten metal.

Remedy-

i. Pattern should have little part as possible in the cope and the most critical surface should be placed in the drag.

6. Wash- Low projection on the drag surface commencing near the surface. 


\section{Cause -}

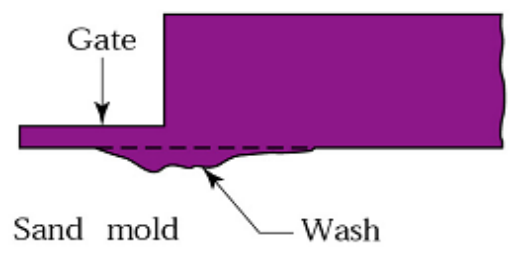

Figure 0-6 wash

i. By the erosion of sand due to high velocity jet of liquid metal in the bottom gate.

ii. Lack of binders in facing and core stand.

iii. Low strength of mould and core.

Remedy-

i. Improve gating system.

ii. $\quad$ Add more binders to facing and core sand.

7. Buckle- Long, fairly shallow, broad, vee- shaped depression occurring in the surface of the flat casting.

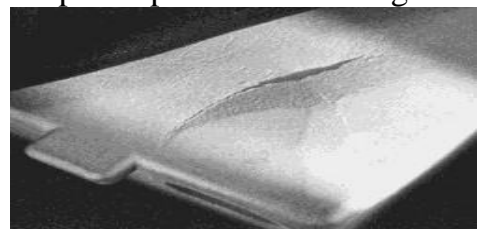

Figure 0-7 Buckle

Cause-

i. Expansion of the thin layer of sand at the mould face takes place before the liquid metal at the mould face solidifies.

Remedy-

i. Proper amount of volatile additives in the sand - mix is essential to make room for this expansion and to avoid buckles.

8. Scab- Rough, thin layer of a metal, protruding above the casting surface on top of a thin layer of sand.

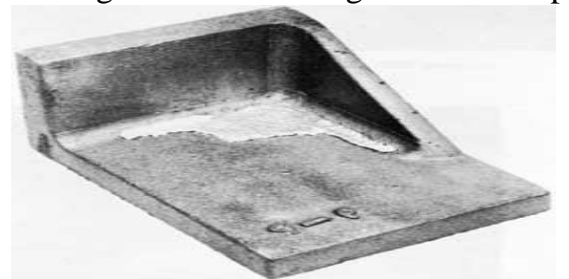

Figure 0-8 Scab

Cause-

i. When the upheaved sand is separated from the mould surface and the liquid metal flows into the space between the mould and the displaced sand.

Remedy-

i. Lower the moisture content of the molding sand, which increases the overall mold strength

ii. Lower the pouring temperature of the metal (eliminate excess superheat), which reduces the amount of sand expansion

iii. Lower the temperature of the molding sand from the return sand system to increase the strength properties of the sand.[3]

9. Penetration- Rough, porous projection on casting. 


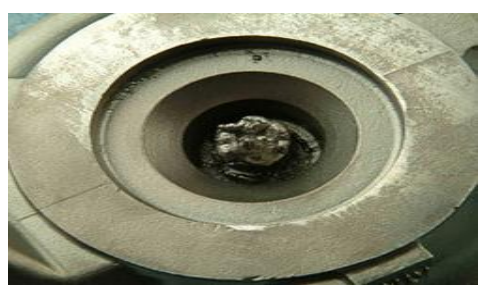

Figure 0-9 Penetration

Cause-

i. If the mould surface is too soft and porous the liquid metal flows between the sand particles up to the distance into the mould.

ii. Large grain size.

iii. Mould sand and core has low strength.

Remedy-

i. $\quad$ Suitable adjustable pouring temperature.

ii. $\quad$ Sand having fine grain size.

iii. Provide hard ramming

10. Swell- Defect is found on the vertical surfaces of a casting.

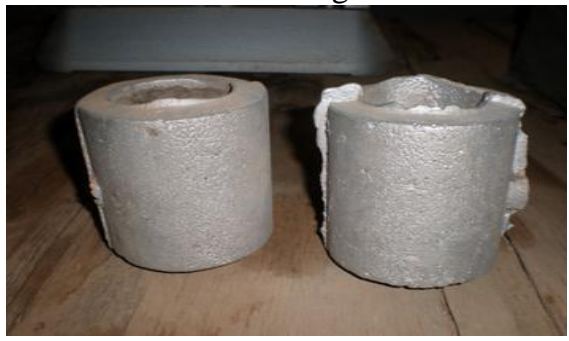

Figure 0-10 Swell

Cause-

i. $\quad$ Deformed by the hydrostatic pressure caused by the high moisture content in the sand.

ii. Improper ramming of the mould.

Remedy-

i. $\quad$ Proper chose of riser.

11. Misrun- Insufficient filling of molten metal in mould cavity.

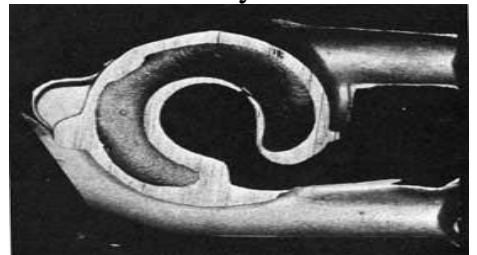

Figure 11.Misrun

Figure 0-11 Misrun

Cause-

i. Due to insufficient superheat, material start freezing before reaching the farthest point of the mould cavity.

ii. Faulty design and gating

Remedy-

i. $\quad$ Adjust proper pouring temperature

ii. Improve design and gating system.

12. Shrinkage- Reduction in required dimension. 
Cause-

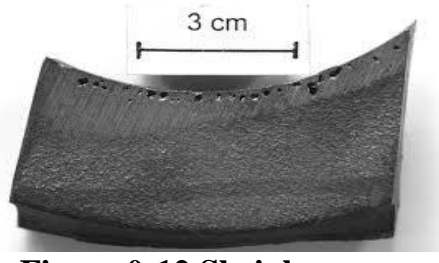

Figure 0-12 Shrinkage

i. $\quad$ Faulty gating and riser system

ii. Improper chilling.

Remedy-

i. Proper directional solidification by modifying gating system, riser and chilling

13. Shift- Misalignment between two halves of the mould.

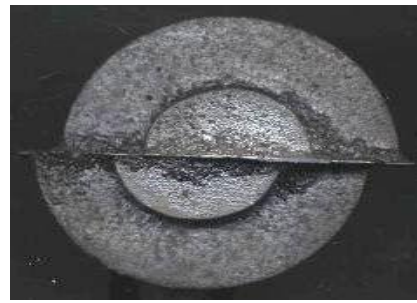

Figure 0-13 Shift

$\begin{array}{ll} & \text { Cause- } \\ \text { i. } & \text { Worn out or bend clamping pin. } \\ \text { ii. } & \text { Misalignment of two halves of patterns. } \\ \text { iii. } & \text { Improper support of core. } \\ & \text { Remedy- } \\ \text { i. } & \text { Repair or replace the support pins. } \\ \text { ii. } & \text { Provide adequate support to core. } \\ \text { iii. } & \text { Increase strength of both mould and core }\end{array}$

14. Porosity- Very small holes uniformly dispersed throughout casting.

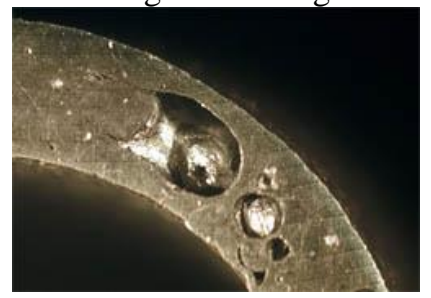

Cause-

Figure 0-14 Porosity

i. Due to decrease in gas solubility during solidification

ii. High pouring temperature

iii. High moisture and low permeability

iv. Less flux used.

Remedy-

i. Control metal composition.

ii. Increase flux proportion

iii. Reduce moisture and increase permeability of mould.

iv. Effective degassing[2]

v.

15. Hot Tear- A crack that develop in casting due to high residual stresses. 
Causes-

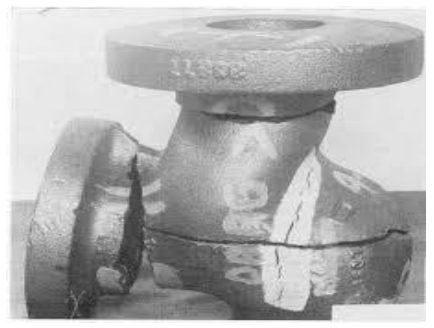

Figure 0-15 Hot Tear

i. $\quad$ Lack of collapsibility in mould

ii. Hard ramming of mould

iii. Lack of collapsibility in core. Remedy-

i. Improve collapsibility of core and mould

ii. Modify casting design

16. Wrap- Distortion due to warp age is known as warp defect.

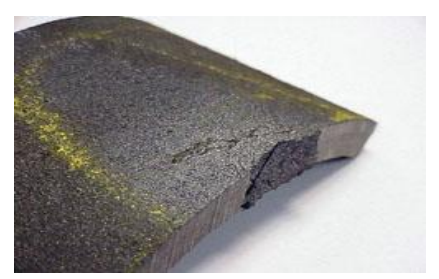

Figure 0-16 Wrap

Causes-

i. Distortion due to warp age can occur over time in casting that partially or completely liberates residual stresses.

Remedy-

i. Common practice in iron casting is normalizing heat treatment to remove residual stress.

17. Flash- Flash is an excess material projecting from casting, generally visible as a thin metallic sheet, perpendicular to the casting face.

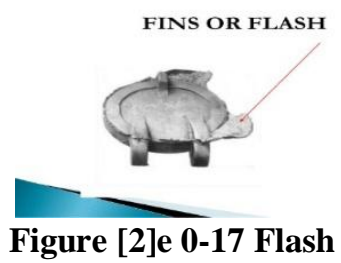

Cause-

i. $\quad$ High pouring temperature.

ii. Pattern having cavities as the end

iii. Improper clamping of top and bottom parts.

Remedy-

i. $\quad$ Ensure end cavities to be filled to avoid metal leakage.

ii. Dimensions to be controlled

iii. Sealing of mould box near parting line

iv. $\quad$ Proper core setting

Process Parameters:-

An Ishikawa diagram (cause and effect diagram, fishbone, herringbone diagram) created by Kaoru Ishikawa that shows cause of a specific event Common uses of the Ishikawa diagram are product design and quality defect prevention to identify potential factors causing an overall effect. Each cause or reason for imperfection is a source of variation. Causes are usually grouped into major categories to identify these sources of variation. 


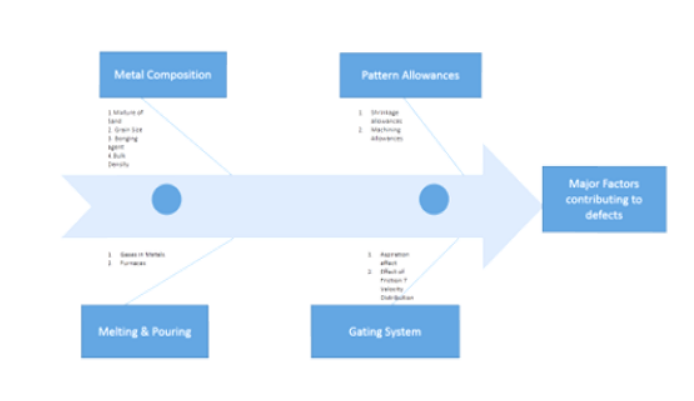

Metal Composition:

Figure 0-1 Cause and Effect diagram

i. The material for a green sand mould is a mixture of 70 to $80 \%$ sand, 10 to $20 \%$ clay, 3 - $6 \%$ water and 1 to $6 \%$ additives. The ration way vary slightly depending on whether the casting is ferrous or nonferrous.

ii. Bulk density of a sand - mix is very low if the grains are of almost equal size with smooth round shape results in an increased void and higher permeability.

iii. Clay together with the water act as a bonding agent and imparts tensile and shear strength to the Moulding sand.

1. Pattern Allowances:

i. Shrinkage allowances should be provided to take care of the contractions of a casting. The total contraction of casting takes places in three stages:-

a. contraction of the liquid from the pouring to freezing temperature.

b. The contraction associated with the change of phase from liquid to solid.

c. The contraction of the solid casting from freezing to room temperature.

ii. Cast surface is too rough to be used in the same way as a result machining allowances are required to produce the finished surface. [1]

2. Melting and Pouring:

i. Specific gases in controlled amount can be beneficial imparting certain desirable qualities to castings.

a. In metal casting the gases may be mechanically trapped

b. May be generated due to the variation in their solubility at different temperatures and phases

c. May be produced due to chemical reaction.

ii. The selection of furnace depends mainly on the metal chemistry, the maximum temperature required and the metal deliver rate and mode.

a. Optimum temperature is decided by a property called fluidity of the metal.

b. The rate and mode of liquid metal delivery are largely decided by the process- batch or continuous melting used.

3. Gating System:

A good gating design ensures distribution of the metal in the mould cavity at a proper rate without excessive temperature loss, turbulence and entrapping gases and slags. The design of a gating system depends on both the metal and mould compositions.

i. $\quad$ Mould made of permeable material care should be taken to ensure the pressure anywhere in the liquid metal stream does not fall below the atmospheric pressure. Otherwise gases originating from baking of the organic compounds. In the mould will enter the molten metal stream, producing porous casting.

ii. The velocity of the fluid in contact with any solid surface is zero and the maximum at the axis of the conduit. The velocity conduit depend on the shape of conduit and the nature of flow. [1]

\section{Conclusion:-}

In this research work different casting defects are studied. By referring different research papers causes and their remedies are listed. These will help to quality control department of casting industries for analysis of casting defect. This study will definitely be helpful in improving the productivity and yield of the casting. Rejections of the casting on the basis of the casting defect should be as minimized and all the above research is heading in the same direction. 


\section{References:-}

1. A. K. M. Amitabha Ghosh, Manufacturing Science, New Delhi: east - West Press Private Limited, 2003.

2. M. R. M. A. B. Dr. D.n.Shivappal, "Analysis of Casting Defects and Identification of Remedial Measure- a Diagnostic study," International Journal of Engineering Inventions ISSN - 2278 - 7461, vol. 1, no. 6, pp. 01 05, October 2012.

3. A. S. Karapse, "Casting Defects Analysis \& Parameters Optimisation through ANOVA," journal of Emerging Technologies \& Innovation Research - JETIR, vol. 3, no. 6, June 2016.

4. A. Juriani, "Casting Defects Analysis in Foundary \& Their Remedial Measures," IOSR - JMCE, vol. 12, no. 6, pp. 43 - 54, Nov. Dec 2015.

5. S. A. N. H. a. H. a. K.M.Karuppannan, "Optimization of Green Sand Casting Process Parameters by using Taguchi,s Method," International journal of Advance Manufacturing Technology , vol. 30, pp. 1040 - 1048, 2006. 